\title{
The Abbreviated Psychopathy Measure (APM): Two Pilot Studies of a Brief Measure of Psychopathy Traits
}

\author{
Robert A. Semel ${ }^{1}$ \\ ${ }^{1}$ Brooklyn, New York, USA \\ Correspondence: Robert A. Semel, Brooklyn, New York, USA \\ Received: April 12, 2018 \\ Accepted: April 28, 2018 \\ Online Published: May 1, 2018 \\ doi:10.5539/ijps.v10n2p41 \\ URL: https://doi.org/10.5539/ijps.v10n2p41
}

\begin{abstract}
Two studies were undertaken to examine preliminary construct validity of a newly developed, abbreviated measure of psychopathy. The Abbreviated Psychopathy Measure (APM) is a 33-item inventory that is closely modeled on the Triarchic Psychopathy Measure (TriPM; Patrick, 2010), with a new and more parsimonious set of items. Analyses in Study $1(N=126)$ found that the Boldness, Meanness, and Disinhibition scales of the APM had high internal consistency reliabilities and were highly correlated with their counterpart scales on the TriPM. The APM Total score was very highly correlated with the TriPM Total score $(r=.90)$. Each of the APM scales was also significantly correlated with a measure of Antisocial Intent. In Study $2(N=140)$, the APM was very highly correlated with the Total score of a 36-item version of the Levenson Self-Report Psychopathy Scale (LSRP; Levenson, Kiehl, \& Fitzpatrick, 1995). Additionally, the APM scales were associated differentially with normal range personality variables associated with psychopathy (e.g., Boldness was robustly associated with Extraversion, Meanness was highly and inversely associated with Agreeableness, Disinhibition was robustly and negatively associated with Conscientiousness). The APM appeared to differ most significantly from the TriPM in that APM Boldness was moderately correlated with Meanness and Disinhibition. APM Boldness may capture a more maladaptive quality of boldness relative to TriPM Boldness through a greater emphasis on low harm avoidance or fearlessness in comparison to TriPM Boldness. The APM is potentially a promising brief measure of psychopathy; however, further study is needed to determine whether the moderately inter-correlated APM scales can distinguish among conceptually relevant constructs. Directions for future research are discussed.
\end{abstract}

Keywords: psychopathy, triarchic model, TriPM, APM, five-factor model of personality

\section{Introduction}

\subsection{Historical and Contemporary Conceptualizations of Psychopathy}

Psychopathy is generally understood as representing a manifestation of interpersonal, affective, and behavioral traits or dimensions (Cleckley, 1941/1976; Cooke \& Michie, 2001; Hare, 1991; 2003; Hare \& Neumann, 2008; Patrick, Fowles, \& Krueger, 2009; Skeem, Polascheck, Patrick, \& Lilienfeld, 2011), as illustrated, for example, by absence of guilt or remorse, callous lack of empathy, superficial charm, shallow affect, lying, deceitfulness, narcissism, manipulation/exploitation of others, poor impulse control, irresponsible, reckless behavior, and anti-social behavior which may include crime and aggression. The study of psychopathy has greatly advanced over the past two decades in both forensic and non-forensic samples (Lilienfeld \& Fowler, 2006; Seibert, Miller, Few, Zeichner, \& Lynam, 2010; Miller \& Lynam, 2015; Miller, Maples-Keller, \& Lynam, 2016). However, there continues to be debate as to what exactly constitutes psychopathy and distinguishes it from other disorders (e.g., Skeem et al., 2011; Miller \& Lynam, 2015; Patrick et al., 2009). There is disagreement over the number of latent dimensions underlying psychopathy. There also is debate whether antisocial behavior is a core feature of psychopathy and whether so-called positive-adjustment indicators as identified by Cleckley (1941/1976), e.g., absence of delusions and other signs of irrational thinking, absence of "nervousness" or psychoneurotic manifestations, are essential to psychopathy. The psychopathic personality has been characterized by some (e.g., McCord \& McCord, 1964) as manifesting hostile alienation, cruelty, aggression and parasitic exploitation. In contrast, Cleckley (1941/1976) did not describe psychopathic patients as persons who are cruel, violent, predatory, or dangerous. The "Behavioral deviance" component in Cleckley's model of psychopathy includes criteria such as "Inadequately motivated antisocial behavior", "Poor judgment and failure to learn by experience", which downplay criminal intention. Hare $(1991,2003)$ developed a model of psychopathy that was based on 
Cleckley's criteria, as reflected in the Psychopathy Checklist-Revised (PCL-R; Hare, 2003). However, unlike Cleckley, who worked with psychiatric patients, Hare developed the PCL-R with criminal samples. Hare's model emphasizes criminal behavior and de-emphasizes positive-adjustment indicators.

Given the somewhat different historical and contemporary conceptualizations of psychopathy, it is not surprising that existing self-report measures of psychopathy involve somewhat different operationalizations of psychopathy (Drislane, Patrick, \& Arsal, 2014). The triarchic model of psychopathy (Patrick et al., 2009) is an integrative model of psychopathy based on essential phenotypic components of psychopathy and is operationalized in the Triarchic Psychopathy Measure (TriPM; Patrick, 2010), which includes the domains Boldness, Meanness, and Disinhibition. Hall et al. (2014) assert that the latter traits may be conceptualized as "open constructs" that can be operationalized by different measures in differing ways. The current study aims to provide preliminary validation of a new self-report measure that is intended to represent the triarchic model with a briefer set of items. Eisenbarth, Lilienfeld, and Yarkoni (2015) noted that abbreviated psychopathy measures lend themselves to the study of large numbers of persons, recruited from college or community samples, and to epidemiological study.

\subsection{The Triarchic Model as an Integrative Model of Psychopathy}

As noted above, the triarchic model of psychopathy reflects an organizing framework or integrative model that may help to reconcile different historical theories and contemporary measurement models through focus on three distinct phenotypic domains of Boldness, Meanness, and Disinhibition. The TriPM (Patrick, 2010) is a 58-item self-report measure designed to assess the triarchic domains. Boldness captures personality qualities such as fearlessness, social dominance, low stress reactivity, emotional resiliency, imperturbability, thrill and adventure seeking. Maladaptive correlates may extend to diminished emotional sensitivity, narcissism, and imperviousness to punishment. Meanness captures callousness, cold-heartedness, antagonism, disdain for and lack of close attachments with others, cruelty, aggression and exploitation of others. Disinhibition captures a propensity toward deficiencies in impulse control, including lack of planfulness and foresight, impaired regulation of affect and urges, demand for immediate gratification and deficient behavioral restraint. Behaviorally this may manifest also as irresponsibility, impatience, impulsive behaviors resulting in negative consequences, alienation and distrust, aggressive acting out, untrustworthiness, proneness to drug and alcohol problems, and illicit and other norm-violating activities (Krueger, Markon, Patrick, Benning, \& Kramer, 2007). The TriPM Meanness and Disinhibition scales were derived from the Externalizing Spectrum Inventory (ESI; Krueger et al., 2007).

\subsection{Empirical Support for Validity of the TriPM}

Considerable support has been developing for construct validity of the TriPM in undergraduate, community, and correctional samples, and in cross-cultural studies (e.g., Drislane et al., 2014; Patrick, 2010; Poy, Segarra, Esteller, Lopez, \& Molto, 2014; Sellbom \& Phillips, 2013; Shou, Sellbom, \& Xu, 2017; Stanley, Wygant, \& Sellbom, 2013). The TriPM has demonstrated medium to large effect size associations with other self-report adult and youth measures of psychopathy and medium effect size estimates with personality measures (Drislane et al., 2014). Drislane et al. (2014) found that the TriPM Disinhibition and Meanness scales were strongly represented in all the adult and youth psychopathy self-report inventories they studied. Boldness was associated with both indices of maladjustment, e.g., rebellious nonconformity, grandiosity, lack of guilt, dishonest charm, and with measures of adaptive function, e.g., wellbeing, achievement, social closeness. The TriPM has also been associated with various measures of personality and psychological adjustment. For example, Blagov, Patrick, Oost, Goodman, and Pugh (2015), found the TriPM subscales to be significantly and meaningfully associated with measures of personality based on the Five-Factor Model of personality (FFM), psychological maladjustment, and personality pathology. When statistically controlling for the other TriPM subscales, Boldness was positively associated with Extraversion, Exhibitionism, and Entitlement, and negatively associated with Neuroticism, Distress, Anxiety, Depression, Low Self-esteem, Low Well-being. The Meanness scale was negatively associated with Agreeableness, Self-restraint, Suppression of Aggression, Consideration of Others, and positively associated with Aggression and Detachment. The Disinhibition scale was negatively associated with Conscientiousness, Self-restraint, Impulse Control, Responsibility, and positively associated with Manipulativeness, Disinhibition, and Impulsivity. Stanley et al. (2013) found TriPM Boldness to be most highly associated with Extroversion and negatively associated with Neuroticism. Meanness was most highly and negatively associated with Agreeableness, and was also significantly and negatively associated with Conscientiousness. Disinhibition was positively associated with Neuroticism and negatively associated with Conscientiousness. Studying three samples of Italian community-dwelling adolescents, Somma, Borroni, Drislane, and Fossati (2016) found that associations between five-factor domain scores and TriPM scale scores were consistent with results that had been found in adults. 
Van Dongen, Drislane, Nijman, Soe-Agnie, and Van Marle (2017) examined the reliability and validity of the TriPM in a Dutch community sample and a Dutch sample of forensic psychiatric patients. As found in other samples, internal consistencies of the TriPM were high in both samples. In both the community and forensic psychiatric patient samples, the TriPM subscales were differentially associated with the higher order factors of the Psychopathic Personality Inventory-Revised (PPI-R; Lilienfeld \& Widows, 2005). Correlations were of moderate to high magnitudes. For example, in both samples, Boldness was highly correlated with Factor 1 of the PPI-R, Fearless-Dominance. The TriPM subscales also were related as expected to measures of reactive and proactive aggression. Additionally, overall the TriPM performed better than the PPI-R in discriminating between the community and forensic samples.

It was demonstrated that Boldness, as indexed by the TriPM, accounted for a significant difference between psychopathy and antisocial personality disorder (Wall, Wygant, \& Sellbom, 2015). Boldness added to the incremental prediction of PCL-R Factor 1 (Interpersonal/Affective) scores above and beyond Antisocial Personality Disorder scores.

A recent meta-analysis by Lilienfeld et al. (2016) found an overall weighted effect size of $r=.39$ (medium to large in magnitude) between Boldness and non-PCL-based psychopathy measures. When the analyses were limited to well-validated psychopathy measures, the weighted correlation increased to .44. Lilienfeld et al. suggest that boldness is integral to the configuration of features that manifest in persons considered to be psychopathic.

It has been demonstrated that items from existing measures can be used to represent each of the triarchic domains. For example, Hall et al. (2014) developed PPI-based Triarchic scales from PPI items relevant to each triarchic facet. The PPI-Triarchic scales demonstrated good internal consistency and relationships with criterion variables consistent with predictions based on the Triarchic model. Brislin, Drislane, Smith, Edens, and Patrick (2015) similarly developed triarchic scales from the Multidimensional Personality Questionnaire (MPQ; Tellegen, 2003), while Drislane et al. (2015) developed triarchic scales from the Youth Psychopathic Traits Inventory (YPI; Andershed, Kerr, Stattin, \& Levander, 2002). These studies lend support to the triarchic model.

As noted by Patrick and Drislane (2014), and by Sellbom, Lilienfeld, Fowler, and McCrary (in press), studies of the TriPM have reported moderate correlations between the Meanness and Disinhibition scales in the range of .4 to .6. Meanness and Boldness scores tend to be more modestly correlated, in the range of .2 to .3. Scores on the Boldness and Disinhibition scales, which are conceptualized as orthogonal dimensions (Patrick et al., 2009), have been found to be minimally correlated or somewhat negatively correlated, ranging from 0 to -2 .

\subsection{Limitations and Questions Regarding Validity of the TriPM}

The conceptual and empirical validity of the TriPM, especially with respect to the role of boldness in psychopathy, have not been without question. Meta-analyses by Marcus, Fulton, and Edens (2013) and by Miller and Lynam (2012) led those researchers to question the role of fearless dominance (which is conceptually very similar to TriPM Boldnes) in the conceptualization of psychopathy, and to conclude that fearless dominance is not essential to psychopathy. Across 61 samples, Miller and Lynam found support for convergent and criterion validity of PPI Factor 2 (Self-Centered Impulsivity-ScI) and for the PPI total score. In contrast, PPI Factor 1 (Fearless Dominance-FD) was most strongly negatively related to negative emotional states, e.g., negative mood, anxiety, and the general internalizing symptom domain, while being positively related to positive emotionality, novelty seeking, and a generally outgoing personality style. FD had a mean weighted $r$ with PCL-R Factor 1 of .23, while the $r$ with PCL-R Factor II was .07. FD manifested small to null relations with measures related to externalizing psychopathology such as aggression, substance use, antisocial behavior, and antisocial personality disorder.

Gatner, Douglas, and Hart (2016) examined the incremental value of TriPM Boldness in understanding antisocial criteria and prosocial criteria. They found that Boldness had small associations with prosocial outcomes, was mostly unrelated to maladaptive outcomes (although weakly correlated with self-reported risky driving behavior), did not display significant interactions with Meanness and Disinhibition to better predict external criteria, generally did not add incrementally to Meanness and Disinhibition, and did not display curvilinear associations with maladaptation. Based on these findings, Gatner et al. suggested that Boldness appears to be more of a marker of positive adjustment and does not appear to be essential to the definition of psychopathic personality disorder.

Gatner, Douglas, and Hart (2017) compared the lexical similarity of the triarchic model of psychopathy to two contemporary models of psychopathy, i.e., the Comprehensive Assessment of Psychopathic Personality (CAPP; Cooke, Hart, Logan, \& Michie, 2012), and the Five Factor Model of psychopathy (FFM; Widiger \& Lynam, 
1998). Lexical similarity indicated the similarity in meaning or definition between two terms or words. The study found that whereas TriPM Meanness and Disinhibition were lexically similar to both the CAPP and FFM psychopathy, Boldness was lexically dissimilar to a large portion of the CAPP and FFM psychopathy. Boldness shared some of its strongest conceptual overlap with FFM facets unessential to psychopathy, such as positive emotions, achievement, activity, warmth, and competence. Gatner et al. concluded that their study provided little evidence to support a central role of Boldness in psychopathy, as defined by the CAPP and FFM.

In a meta-analysis of the relationships between the Dark Triad (Machiavellianism, Narcissism, and Psychopathy) and the five-factor model of personality, O'Boyle, Forsyth, Banks, Story, and White (2015), using relative importance analyses, found that $63 \%$ of the FFM-explained variance in psychopathy scores was accounted for by Agreeableness, followed by $27 \%$ for Conscientiousness. Extraversion and Neuroticism (Boldness has been associated with high Extraversion and low Neuroticism) explained $8 \%$ and $1 \%$, respectively, of the meta-analyzed psychopathy scores.

As noted by Evans and Tully (2016), excitement seeking on the TriPM is included in the context of meanness whereas it may be more indicative of boldness. The TriPM conceptualizes psychopathy as reflecting distinguishable facets rather than reflecting a unitary or higher order construct of psychopathy. Yet, as noted earlier, moderate relationships have been reported between the Meanness and Disinhibition scales. Evans and Tully suggest that these correlations might be explained by multidimensionality of the TriPM scales, or by intersecting components of the constructs; it is not fully clear whether such overlap is an issue related to validity. Sellbom and Phillips (2013) found unexpected significant relationships between meanness with sensation seeking, disinhibition and boredom susceptibility. This result led Evans and Tully to conclude that TriPM meanness appears to also be measuring disinhibitory tendencies. As such, it appears that some elements of the TriPM scales have not been found to discriminate between distinct facets or constructs.

Notwithstanding potential limitations or unresolved debates concerning the triarchic model and the TriPM measure, it appears that the TriPM will continue to contribute significantly to the understanding and assessment, and possibly to treatment approaches, of psychopathy. However, at present no alternative, briefer triarchic scales are available.

\subsection{Purpose of Current Studies}

A new scale was developed by this author with the intention of assessing the triarchic domains with a reliable, valid, and more parsimonious set of items. The scale is tentatively titled the Abbreviated Psychopathy Measure (APM). A shorter measure that indexes the triarchic domains would be economical in research contexts, and is thus consistent with the advantages of the self-report method of assessment of psychopathy (Lilienfeld \& Fowler, 2006). More generally with respect to psychological assessment of personality traits, more concise scales not only are time saving but help to avoid or reduce boredom and fatigue (Burisch, 1984, 1997; Saucier, 1994).

Study 1: The primary aim of this study was to demonstrate that the APM would be a reliable measure that is significantly associated with the TriPM and that the respective triarchic scales from each measure would be more highly correlated with each other than with the remaining triarchic scales, e.g., APM Boldness should be most highly correlated with TriPM Boldness. A secondary aim was to demonstrate that the APM would demonstrate significant association with an external correlate, i.e., a measure of antisocial intent. It was further anticipated that the APM Disinhibition scale would have the strongest association with Antisocial Intent.

Study 2: To further explore and to validate the APM by examining the relationships between the APM scales and, a) another brief psychopathy measure; and b) normal-range personality variables. It was predicted that the APM Total score would be highly correlated with the Total score of the 36-item, three-factor Levenson Self-Report Psychopathy Scale (LSRP; Levenson, Kiehl, \& Fitzpatrick, 1995) that was developed by Christian and Sellbom (2016). It was also predicted that APM Meanness and Disinhibition would be most highly correlated with LSRP Callous and Antisocial subscales, respectively. Secondly, the current study examined whether the APM scales would be differentially related to normal range personality variables in a pattern similar to what has been found with the TriPM and the five-factor model of personality (e.g., Poy et al., 2014; Stanley et al., 2013; Somma et al., 2016). Specifically, it was expected that Boldness would be significantly and positively correlated with Extraversion and negatively correlated with Neuroticism. Meanness was expected to be significantly negatively correlated with Agreeableness and to a lesser extent with Conscientiousness. Disinhibition was expected to be negatively correlated with Conscientiousness, and to a lesser extent with Agreeableness. Disinhibition also was expected to be positively correlated with Neuroticism. 


\section{Study 1}

\section{Method}

\subsection{Participants}

Participants were recruited through Amazon Mechanical Turk (MTurk), an online marketplace in which "workers" perform Human Intelligence Tasks (HITS) for "requesters" for the completion of computerized tasks. MTurk has become increasingly popular as a resource for behavioral research data among social scientists (Paolacci \& Chandler, 2014). Participants included 74 men (59\%) and 52 women (41\%) between the ages of 21 and 69 years, with a mean age of $33.8(S D=9.8)$.

\subsection{Procedure}

Participants recruited from MTurk were directed to a link at SurveyMonkey where they were provided a brief description of the study and elements contained in the framework for informed consent, such as expected length of time, potential risks, discomforts, benefits, voluntary nature of study, confidentiality, and contact information. Participants provided their consent on the survey form prior to answering the remainder of the survey. Consent was recorded electronically together with the participants' survey responses. Participants were paid through MTurk for their participation. Following several demographic items, the 33 items of the APM were presented, followed by the 58 items of the TriPM Inventory, and the 12 items of the Antisocial Intent scale from the Measures of Criminal Attitudes and Associates (MCAA). All participants received the same order of items.

\subsection{Measures}

The Abbreviated Psychopathy Measure (APM) (see Table 1) in its current form is a 33-item self-report scale designed to assess the constructs of Boldness, Meanness, and Disinhibition as postulated by Patrick et al. (2009). Five of the 11 items included in the APM Meanness scale were selected from the Caring-Uncaring Emotional (CUE) Inventory (Semel, 2016, 2017). The CUE Inventory was designed to measure affective traits of psychopathy. In two studies of community samples of adults (Semel, 2016, 2017), the CUE was found to have high internal consistency reliability ( $\alpha=.91$ and .93 , respectively). The CUE was found to have high correlations with an expanded, 36-item version of the Levenson Self-Report Psychopathy Scale (LSRP; Levenson, Kiehl, \& Fitzpatrick, 1995) that was developed by Christian and Sellbom (2016), and with multiple subscale measures of callous affect from several established adult and youth psychopathy measures. The CUE was highly correlated with the TriPM Meanness scale $(r=.84)$. Exploratory Factor Analysis in both studies identified a first factor that appeared to represent a robust measure of callousness. Items from the CUE Callousness subscale that were selected for the APM Meanness were items that had higher loadings on the EFA's in the two CUE studies. Six new items were added for a total of 11 items in the APM Meanness scale. An example of items from the Meanness scale includes the item "People might describe me as mean and cruel". Items for the APM Boldness and Disinhibition scales were developed to reflect their respective behavioral characteristics as described by Patrick et al. (2009) and Patrick (2010). For example, one item on the Boldness scale ("Situations that others might see as dangerous I would see as exciting") is intended to capture tolerance for danger and thrill-adventure seeking. One item on the Disinhibition scale ("If I see a chance to take something I want, I take it, whether it's legal or not") is intended to capture the propensity toward impulse control problems and impaired regulation of urges. The response format for the APM items utilizes a 4-point Likert-type scale that included the choices "Disagree strongly", "Disagree somewhat", "Agree somewhat", "Agree strongly". Scores range from 1 to 4 . All items on the APM are keyed so that higher scores reflect higher levels of their respective dimensions. 
Table 1. Abbreviated Psychopathy Measure

\section{Items}

\section{Boldness}

1. Situations that others might see as dangerous I would see as exciting.

2. My fearless and stress-free personality would help me to be good at being an undercover agent or spy.

3. I enjoy competition, and I always expect to win.

4. I prefer safety and security rather than daring and adventure.

5. I have a very strong and dominating personality.

6. I always stay incredibly cool and calm no matter what the situation.

7. I can persuade anyone to go along with my ideas.

8. I am willing to take great risks in life without fear of loss or failure.

9. What's the fun in life unless you're willing to face a little danger.

10. I could be a star in a reality TV adventure show like "Survivor".

11. Among my talents, I am excellent at being a leader and organizer.

\section{Meanness}

12. People might describe me as mean and cruel.

13. I can be good at pretending to care about people, but most of the time I really don't care.

14. If there is someone I don't like, it would feel good to see them get hurt.

15. I really care about how I treat other people.

16. Some people might describe my actions as aggressive and harmful.

17. I am good at taking advantage of people's weaknesses.

18. I don't spend my time worrying about people's feelings getting hurt.

19. It would be very hard for me to be cold and heartless to people.

20. I could look people straight in the eye, and it means nothing to me to lie or to cheat them.

21. It sometimes gives me pleasure to see someone in pain.

22. I might not say this to others, but people basically are like objects for me to use to get what I want.

\section{Disinhibition}

23. I'm the type of person to act quickly without thinking it through which sometimes gets me in trouble.

24. If I see a chance to take something I want, I take it, whether it's legal or not.

25 . The saying "plan ahead" is definitely not for me.

26. I almost always put on the brakes before I act out on my impulses.

27. Playing by the rules sounds nice, but getting what I want is more important.

28. Some people might describe my actions as being unpredictable.

29. I have stolen my share of things; the trick is just not to get caught.

30. So what if I have broken lots of promises, people shouldn't take things so seriously.

31. I admit that some of my family or friends think I act recklessly.

32. I often find myself in trouble before I even know what I did wrong.

33. I am very cautious not to act before I think about the consequences.

Triarchic Psychopathy Measure (TriPM). The TriPM (Patrick, 2010) is a nonproprietary 58-item self-report inventory designed to measure the three phenotypic psychopathy constructs of Boldness, Meanness, and Disinhibition. The Boldness and Meanness scales each consist of 19 items while the Disinhibition scale consists of 20 items. The TriPM has demonstrated medium to large effect size associations with other self-report measures of psychopathy and medium effect size associations with personality measures (Drislane et al., 2014). 
The Meanness and Disinhibition scales tend to be moderately correlated, while the Meanness and Boldness scales tend to be more modestly correlated. The Boldness and Disinhibition scales tend to be minimally correlated (see Sellbom et al., in press). The TriPM utilizes a 4-point Likert-type response choice, i.e., "False", "Mostly False", "Mostly True", "True", with scores ranging from 0 to 3. Some studies use score ranges of 1 to 4.

The Measures of Criminal Attitudes and Associates (MCAA). The MCAA (Mills, Kroner, \& Forth, 2002) is a two-part, self-report measure of antisocial attitudes and antisocial associates. In the current study, only the 12-item Antisocial Intent scale was used, with response choices ranging from "Strongly disagree" to "Strongly agree". A 7-point Likert-type scale was used, including a midpoint choice of "Neither agree nor disagree", to increase the variance at the extremes of the distribution. The MCAA has demonstrated significant associations with other measures of antisocial attitudes and criminal history indices (Mills et al., 2002), and has demonstrated predictive validity for the outcomes of general and violent recidivism in a sample of male adult offenders (Mills, Kroner, \& Hemmati, 2004). As reported by Mills et al. (2002), in a student sample the MCAA was significantly correlated with other measures of antisocial attitudes and with self-reported antisocial behavior. In the study by Semel (2017), the MCAA Antisocial Intent scale was found to have high internal consistency reliability ( $\alpha$ $=.92$ ).

\section{Results}

\subsection{Descriptive Statistics}

Means, standard deviations, and internal consistencies are shown in Table 2. All measures had high internal consistency coefficients and reasonable mean inter-item correlations. Despite the APM being composed of shorter scales relative to the TriPM, alpha coefficients were quite comparable in comparison to the TriPM scales. Mean inter-item correlation was higher for the APM Total than for the TriPM Total. It is noted that in both the APM and the TriPM, the mean score on the Boldness scale is higher than the mean scores of the Meanness and Disinhibition scales. Except for the APM Disinhibition scale (in which men's scores were marginally higher than women's scores, $p=.06$ ), men scored significantly higher than women on each of the remaining APM scales, on the TriPM scales, and on the Antisocial Intent scale. Age was significantly and negatively associated with all the APM scales and with the TriPM scales except for the Boldness scale. Effect sizes were of small magnitudes. Age was also negatively associated with the Antisocial Intent scale, but of a small magnitude.

Table 2. Means (M), Standard Deviations (SD) and Internal Consistency Coefficients ( $\alpha$ ) of All Measures

\begin{tabular}{llll}
\hline Scale & M & SD & $\alpha($ MIC) \\
\hline APM Bold & 25.33 & 7.11 & $.90(.44)$ \\
APM Mean & 18.56 & 6.71 & $.90(.44)$ \\
APM Dis & 18.88 & 6.32 & $.90(.44)$ \\
APM Total & 62.77 & 16.91 & $.94(.33)$ \\
TriPM Bold & 30.33 & 10.21 & $.90(.31)$ \\
TriPM Mean & 12.30 & 10.10 & $.92(.39)$ \\
TriPM Dis & 12.60 & 9.68 & $.91(.35)$ \\
TriPM Total & 55.22 & 21.43 & $.93(.19)$ \\
MCAA & 34.32 & 15.66 & $.91(.45)$
\end{tabular}

Note. APM $=$ Abbreviated Psychopathy Measure $(11$ items each in the Boldness, Meanness, and Dis $=$ Disinhibition scales). TriPM $=$ Triarchic Psychopathy Measure (19 items each in the Boldness and Meanness scales and 20 items in the Disinhibition scale). MCAA $=$ Measures of Criminal Attitudes and Associates (Antisocial Intent scale; 12 items). MIC, Mean inter-item correlation.

\subsection{Concurrent Validity}

It was expected that the APM would be significantly associated with the TriPM and that the respective triarchic scales from each measure would be more highly correlated with each other than with the remaining triarchic scales. As seen in Table 3, a very high correlation was found between the APM Total and TriPM Total scales ( $r$ $=.90$ ), indicating an overall strong correspondence between these measures. Each APM scale was highly correlated with its counterpart TriPM scale. Additionally, each of the APM scales was more highly correlated with its counterpart on the TriPM than with the remaining two triarchic scales, albeit, the APM Disinhibition 
scale was highly correlated with both the TriPM Disinhibition scale $(r=.85)$ and the TriPM Meanness scale $(r$ $=.77)$.

Table 3. Zero Order Correlations Between All Study Measures

\begin{tabular}{|c|c|c|c|c|c|c|c|c|c|}
\hline Scale & 1 & 2 & 3 & 4 & 5 & 6 & 7 & 8 & 9 \\
\hline 1 APM Bold & - & & & & & & & & \\
\hline 2 APM Mean & $.43 *$ & - & & & & & & & \\
\hline 3 APM Dis & $.51^{*}$ & $.74 *$ & - & & & & & & \\
\hline 4 APM Total & $.79 *$ & $.86^{*}$ & $.88^{*}$ & - & & & & & \\
\hline 5 Tri Bold & $.73 *$ & .07 & .11 & $.38^{*}$ & - & & & & \\
\hline 6 Tri Mean & $.51^{*}$ & $.84 *$ & $.77 *$ & $.83^{*}$ & .15 & - & & & \\
\hline 7 Tri Dis & $.33 *$ & $.70^{*}$ & $.85^{*}$ & $.73 *$ & -.08 & $.74 *$ & - & & \\
\hline 8 Tri Total & $.74 *$ & $.74 *$ & $.80^{*}$ & $.90^{*}$ & $.51^{*}$ & $.88^{*}$ & $.76^{*}$ & - & \\
\hline 9 MCAA & $.42 *$ & $.69 *$ & $.70^{*}$ & $.71 *$ & .14 & $.69^{*}$ & $.62 *$ & $.67 *$ & - \\
\hline
\end{tabular}

Note. ${ }^{*} p<.001$.

Whereas in previous studies the TriPM Meanness and Disinhibition scales have demonstrated moderate correlations between .4 and .6, in the current study their correlation was .74. The correlation between the APM Meanness and Disinhibition scales also was .74, which indicates that in this sample there was a strong overlap, approximately $55 \%$ shared variance, between the Meanness and Disinhibition scales on both the APM and the TriPM.

\subsection{Criterion Validity}

As anticipated, the APM was significantly associated with the Antisocial Intent scale of the MCAA. Of the triarchic scales, APM Disinhibition had the highest correlation with Antisocial Intent, exceeded only by correlation between the APM Total score and the MCAA $(r=.71)$. It is noted that the APM Disinhibition scale had a somewhat higher correlation with Antisocial Intent in comparison to the association between TriPM Disinhibition and Antisocial Intent.

\subsection{Differences between Scale Configurations on the APM and the TriPM}

A notable difference between the configuration of inter-scale relationships on the APM and on the TriPM is that the APM Boldness scale was found to be moderately correlated with both the APM Meanness and Disinhibition scales. In contrast, the TriPM Boldness scale had a low correlation with the TriPM Meanness scale $(r=.15)$. The TriPM Boldness and Disinhibition scales had a low, inverse association $(r=-.08)$. These correlations, though, are consistent with findings in other studies with the TriPM (see Patrick \& Drislane, 2014). Thus, it may be that the relatively less strong association between the APM Boldness scale and the TriPM Boldness scale, as well as the moderately strong associations between the APM Boldness scales and the remaining APM scales, suggests that the APM Boldness scale may in part be tapping into a somewhat different construct (in comparison to the TriPM Boldness scale), or that the APM Boldness scale may be capturing a more maladaptive quality of boldness. This will be expanded upon in the next section.

\subsection{Item Endorsement Frequency}

An examination of item endorsement frequencies reveals differences between the APM and the TriPM, particularly on their respective Boldness scales. On the APM Boldness scale, for four of 11 items (36\%), a majority of the participants endorsed the response that was in the direction of being higher on the dimension of boldness. For example, the item "Among my talents, I am excellent at being a leader and organizer" was endorsed "Agree Somewhat" or "Agree Strongly" by $60 \%$ of respondents. The item "I enjoy competition, and I always expect to win" was endorsed "Agree Somewhat" or "Agree Strongly" by $56 \%$ of respondents. In contrast, on the APM Meanness and Disinhibition scales, none of the items received a majority endorsement in the direction of being higher on their respective dimensions (of psychopathy).

On the TriPM Boldness scale, on 12 of 19 items $^{1}$ (63\%), the majority of participants endorsed a response that was in the direction of being higher on the dimension of boldness. For example, on the items "I' $m$ optimistic more often than not", and "I am well-equipped to deal with stress", $77 \%$ of participants responded "Mostly True" or "True". For the item "I don't stack up well against most others", $72 \%$ of participants responded 
"False" or "Mostly False". Thus, in this study sample, irrespective of reverse-coded items, for the majority of items on the TriPM Boldness scale, a majority of participants responded in the direction of higher boldness. Results that were obtained with a larger sample on the TriPM $^{2}$ reflected a more even split between items endorsed in the direction of higher boldness and items endorsed in the direction of lower boldness. However, in both the current sample and the previous larger sample, the mean score on the set of Boldness items identified as Bold Adaptive was significantly higher $(p<.0001)$ than the mean score on the set of Boldness items identified as Bold Maladaptive. On neither the TriPM Meanness or Disinhibition scales did the majority of participants endorse responses in the direction of higher psychopathy dimensionality. However, on the Disinhibition scale item "I often act on immediate needs", the single most frequent response was "Mostly True" (40\%), albeit a slight majority disagreed in their response to the item (although in a larger sample obtained by this author the majority of respondents agreed with this item).

\section{Discussion}

The Abbreviated Psychopathy Measure (APM) was developed to index the triarchic psychopathy domains with a more parsimonious set of items. Results of this study found the APM scales to have high internal consistency reliabilities and each scale was highly correlated with its counterpart scale on the Triarchic Psychopathy Measure (TriPM). A very high correlation was seen between the APM Total and TriPM Total scales indicating an overall strong correspondence between these measures, in support of concurrent validity of the APM. As anticipated, each of the APM scales was more highly correlated with its counterpart scale on the TriPM than with the remaining two triarchic scales, albeit, the APM Disinhibition scale was highly correlated with both the TriPM Disinhibition scale and with the TriPM Meanness scale. Also, the APM Meanness and Disinhibition scales were more highly correlated than is desirable in scales intended to discriminate between related but also distinct constructs. However, the TriPM Meanness and Disinhibition scales were also highly correlated (at the same magnitude as APM Meanness and Disinhibition), and were more highly correlated in this study as compared to other published reports. Poy et al. (2014) reported a moderate to high correlation between TriPM Meanness and Disinhibition $(r=.62)$; Fanti, Kyranides, Drislane, Colins, and Andershed (2016), reported a correlation of .59 between Meanness and Disinhibition; and Kyranides, Fanti, Sikki, and Patrick (2017), reported a correlation of .66 between Meanness and Disinhibition. However, other studies (e.g., Stanley et al., 2013; Wall et al., 2015) have reported more moderate relationships between these scales. Thus, the magnitude of association between the Meanness and Disinhibition scales in both the APM and the TriPM may be somewhat sample specific, and might be found to be lower in other samples. However, the mean scores on the TriPM in this sample were quite similar to those reported by Van Dongen et al. (2017) in a Dutch community sample, suggesting that the current sample is probably not very different from other community samples in its response to the TriPM, although the mean score on Disinhibition tends to be higher relative to Meanness in other published reports (e.g., Poy et al., 2014; Shou et al., 2017; Somma et al., 2016; Stanley et al., 2013; Wall et al., 2015). Further study will be needed to determine whether, in its current form, the APM Disinhibition scale can discriminate between the Meanness and Disinhibition constructs as theorized in the triarchic psychopathy model. As noted earlier, there is some question whether the TriPM Meanness and Disinhibition scales are truly indexing distinct constructs. At the same time, of the six triarchic scales among the APM and TriPM, the APM Disinhibition scale had the highest correlation with a measure of Antisocial Intent. It is noted that the association between APM Disinhibition and Antisocial Intent was only marginally greater than the associations between the respective Meanness scales of the APM and TriPM with Antisocial Intent. Still, there appears to be a robust association between Disinhibition, as assessed by the APM Disinhibition scale, and antisocial intent/attitudes. This supports criterion validity of the APM, although there is shared method variance between these self-report measures which may have inflated the associations.

The APM Boldness scale appears to potentially tap into a more maladaptive or less socially approved quality of Boldness relative to the Boldness construct as indexed by the TriPM. This was seen in the moderately overlapping relationships between APM Boldness and the APM Meanness and Disinhibition scales. APM Boldness also had a strong correlation with the APM Total, whereas TriPM Boldness had a moderate relationship with TriPM Total. APM Boldness had a moderate correlation with Antisocial Intent. TriPM Boldness had a low, nonsignificant association with Antisocial Intent. Additionally, in comparison to TriPM Boldness, APM Boldness had a much lower percentage of items in which the majority of participants endorsed items in the direction intended to be higher on the boldness dimension. It is possible that the APM Boldness scale is more sensitive, relative to TriPM Boldness, to a more maladaptive quality of boldness which resulted in greater overlap with meanness and disinhibition. It is also conceivable that in part the apparent orthogonal relationship between TriPM Boldness and Disinhibition results from more socially favored item content in the 
former scale. It is also noted that whereas the TriPM Boldness scale is composed of 10 reverse-coded items, the APM Boldness has only one reverse-scored item. Thus, the APM Boldness scale might be more susceptible to a method factor. However, the current study suggests that the TriPM Boldness scale might also be influenced by a method factor in that approximately half of the items were endorsed in the keyed direction of being higher on Boldness by a majority of study participants. Thus, it appears that approximately half of the TriPM Boldness items may be viewed as socially favored or adaptive, whereas approximately half of the items may be viewed as more socially deviant.

A recent study of the TriPM in a Chinese sample by Shou et al. (2017) found the Boldness scale to be multidimensional, with two subscales capturing emotional stability and social dominance. Items on the APM Boldness scale appear to reflect fearlessness, risk-taking, and adventure seeking, but with several items reflecting social potency. It may be that the APM Boldness scale has greater emphasis on lack of harm avoidance that can overlap with impulsiveness - perhaps a willingness to flirt with danger can border on recklessness or impulsiveness - while not reflecting social potency as well as the TriPM Boldness scale. In any case, the moderately high correlations between APM Boldness with Meanness and Disinhibition raises question whether APM Boldness can distinguish between relevant constructs. Whether the APM Boldness scale may be reflecting a more maladaptive quality of boldness in comparison to TriPM Boldness and whether it can distinguish between relevant constructs will need to be further studied.

\section{Study 2}

\section{Method}

\subsection{Participants}

Participants $(N=140)$ were recruited through Amazon Mechanical Turk (MTurk), and included 84 men (60\%) and 56 women $(40 \%)$ with a mean age of $34.2(S D=9.8)$.

\subsection{Procedure}

Participants recruited from MTurk were directed to a link at SurveyMonkey where they were provided a brief description of the study and elements contained in the framework for informed consent, such as expected length of time, potential risks, discomforts, benefits, voluntary nature of study, confidentiality, and contact information. Participants provided their consent on the survey form prior to answering the remainder of the survey. Consent was recorded electronically together with the participants' survey responses. Participants were paid through MTurk for their participation. Following several demographic items, the 33 items of the APM were presented, followed by the 36 items of the expanded LSRP, followed by the 44 items of the Big Five Inventory. All participants received the same order of items.

\subsection{Measures}

The Abbreviated Psychopathy Measure (APM) in its current form is a 33-item self-report scale designed to assess the constructs of Boldness, Meanness, and Disinhibition as postulated by Patrick et al. (2009). This measure was described in greater detail in Study 1.

The expanded 36-item, three factor model of the Levenson Self-Report Psychopathy Scale, as reported by Christian and Sellbom (2016), consists of 11 items composing the Egocentric subscale, 12 items composing the Callous subscale, and 13 items composing the Antisocial subscale. Internal consistency reliability coefficients were reported as .90 for the Total scale, .85 for the Egocentric subscale, .80 for the Callous subscale, and .81 for the Antisocial subscale. The response format utilizes a 4-point Likert-type scale that includes the choices "Disagree strongly", "Disagree somewhat", "Agree somewhat", and "Agree strongly". Scores range from 1 to 4.

The Big Five Inventory (BFI; John, Donahue, \& Kentle, 1991) is a 44-item screening measure of the five-factor model (John \& Srivastava, 1999). The big five dimensions include extraversion vs. introversion, agreeableness vs. antagonism, conscientiousness vs. lack of direction, neuroticism vs. emotional stability, and openness vs. closedness to experience. Items are rated on a 5-point, Likert-type scale ranging from 1 (Disagree strongly) to 5 (Agree strongly).

\section{Results}

\subsection{Concurrent Validity: Associations between APM Scales and LSRP Scales}

Zero-order correlations between the APM scales and the LSRP scales are presented in Table 4. As anticipated, the APM Total scale was very highly correlated with the LSRP Total scale $(r=.88, p<.001)$, reflecting a strong correspondence, overall, between these measures. The APM Boldness scale was most highly correlated with the LSRP Egocentric scale, suggesting noteworthy overlap between these scales. In contrast to what was anticipated, 
the APM Meanness scale was slightly more highly correlated with the LSRP Egocentric scale than it was with the LSRP Callous scale, albeit, it is noted that the LSRP Callous and Egocentric scales were highly intercorrelated. The APM Disinhibition scale had its highest correlation with the LSRP Antisocial scale, but was also highly correlated with the LSRP Egocentric scale. The LSRP Egocentric and Antisocial scales were themselves highly intercorrelated. The Antisocial subscale had its strongest association with APM Disinhibition, and the Callous subscale had its strongest association with APM Meanness, while the Egocentric subscale also had its strongest association with APM Meanness. Thus, there were some conceptually consistent differential associations between the APM scales and the three-factor LSRP scales.

Table 4. Zero Order Correlations between APM Scales and Three-Factor LSRP Scales

\begin{tabular}{lllllllll}
\hline Scale & 1 & 2 & 3 & 4 & 5 & 6 & 7 & 8 \\
\hline 1 Bold & & & & & & & & \\
2 Mean & $.54^{*}$ & & & & & & \\
3 Dis & $.57^{*}$ & $.71^{*}$ & & & & & \\
4 APM Total & $.83^{*}$ & $.87^{*}$ & $.88^{*}$ & & & & \\
5 Egocentric & $.58^{*}$ & $.81^{*}$ & $.77^{*}$ & $.83^{*}$ & & & \\
6 Callous & $.35^{*}$ & $.77^{*}$ & $.51^{*}$ & $.63^{*}$ & $.64^{*}$ & & \\
7 Antisocial & $.49^{*}$ & $.66^{*}$ & $.84^{*}$ & $.76^{*}$ & $.67 *$ & $.41^{*}$ & \\
8 LSRP Total & $.57^{*}$ & $.87^{*}$ & $.85^{*}$ & $.88^{*}$ & .92 & $.78^{*}$ & $.84^{*}$ & \\
\hline Note. ${ }^{*} p<.001$ & & & & & & &
\end{tabular}

\subsection{Associations Between APM Scales and Normal Range Personality Variables}

Zero-order correlations between the APM scales and normal-range personality traits from the Big Five Inventory are presented in Table 5. APM Boldness was robustly associated with Extraversion, modestly and negatively associated with Neuroticism and more modestly and positively associated with Openness. APM Meanness was robustly and negatively associated with Agreeableness and more moderately and negatively associated with Conscientiousness, and, to a lesser degree, was positively associated with Neuroticism. APM Disinhibition had a robust negative association with Conscientiousness, and was more moderately and negatively associated with Agreeableness. Disinhibition was not significantly associated with Extraversion or Openness. Disinhibition was only modestly positively associated with Neuroticism. However, given the moderate correlation between the APM Boldness and Disinhibition scales, partial correlations were performed to examine the relationships between Boldness and Neuroticism when controlling for Disinhibition, and between Disinhibition and Neuroticism when controlling for Boldness. A moderate, negative relationship was seen between Boldness and Neuroticism when controlling for Disinhibition $(r=-.46, p<.001)$. A moderate, positive association was seen between Disinhibition and Neuroticism when controlling for Boldness $(r=.44, p<.001)$. The relationship between Meanness and Neuroticism became nonsignificant $(r=.10, p=.24)$ when controlling for Disinhibition.

Table 5. Correlations Between APM Scale Scores and Normal-Range Personality Variables

\begin{tabular}{llll}
\hline Scale & Boldness & Meanness & Disinhibition \\
\hline BFI & $r$ & $r$ & $r$ \\
Neuroticism & & & \\
Extraversion & $-.26^{*}$ & $.21^{*}$ & $.20^{*}$ \\
Openness & $.57^{* *}$ & .01 & .10 \\
Agreeableness & $.22^{*}$ & -.16 & -.04 \\
Conscientiousness & -.05 & $-.67^{* *}$ & $-.34^{* *}$ \\
\hline
\end{tabular}

Note. $\mathrm{BFI}=$ Big Five Inventory. ${ }^{*} p<.02 . * * p<.001$.

\section{Discussion}

This study had a two-fold aim to further examine validity of the APM by demonstrating an association between the APM and another brief psychopathy measure, and by demonstrating differential associations between the APM scales with normal range personality variables from the five-factor model. The APM was found to have a 
very strong association, overall, with the expanded, three-factor LSRP, adding to concurrent validity of the APM. Despite the moderate to strong correlations within the APM scales and the LSRP subscales, some marginal differential associations between the APM scales and LSRP subscales were noted, e.g., the strongest association was between the LSRP Antisocial subscale and the APM Disinhibition scale. This is consistent with the study by Christian and Sellbom (2016) who found the strongest association between the TriPM scales and the expanded LSRP was that between TriPM Disinhibition and the LSRP Antisocial subscale. Results of the current study differed greatly from those of Christian and Sellbom in that APM Boldness was robustly associated with the Egocentric and Antisocial subscales and modestly associated with the Callous subscale. In contrast, Christian and Sellbom found that TriPM Boldness was not significantly associated with the Egocentric subscale, while it was modestly associated positively with the Callous subscale and modestly associated negatively with the Antisocial subscale. That is, higher TriPM Boldness scores were associated with both lower Antisocial scores and higher Callous scores. Christian and Sellbom interpreted the significant positive association between Boldness and the Callous subscale as possibly reflecting some drift in the Callous subscale toward the Boldness aspect of psychopathy. An alternative possible explanation is that there may be some drift of Boldness toward Callousness, as suggested, for example, by the findings of Drislane et al. (2014), in which Boldness significantly predicted scores on the Callous Affect scale of the SRP-III.

As noted earlier, multiple studies have found similarities in the associations between the TriPM and normal range personality variables. For example, Agreeableness vs. Antagonism has been mostly strong associated with the Meanness scale of the TriPM. Boldness has been robustly (positively) associated with Extraversion and negatively associated with Neuroticism. The current study of the APM demonstrated several similarities to such previous studies with the TriPM. For the APM Boldness, Meanness, and Disinhibition scales, the differential associations with five-factor domains were similar to what has been reported in studies with the TriPM (e.g., Blagov et al., 2015; Patrick \& Drislane, 2014; Poy et al., 2014). The magnitude of associations between APM Boldness and (BFI) Extraversion, and between APM Meanness and (BFI) Agreeableness, and between APM Disinhibition and (BFI) Conscientiousness were quite similar to what has been reported in studies with the TriPM. Despite the high correlation between APM Meanness and Disinhibition, APM Meanness had a much greater (negative) association with Agreeableness than did APM Disinhibition, while APM Disinhibition had a considerably greater (negative) association with Conscientiousness than did APM Meanness. In contrast, Boldness had a modest, inverse association with Neuroticism, while Disinhibition had a modest, positive association with Neuroticism. However, a moderate, negative relationship was seen between Boldness and Neuroticism when controlling for Disinhibition $(r=-.46, p<.001)$, and a moderate, positive association was seen between Disinhibition and Neuroticism when controlling for Boldness $(r=.44, p<.001)$. The magnitudes of these associations are quite like what has been reported in studies of the TriPM and normal range personality variables. Poy et al. (2014) found the correlation between Neuroticism and Disinhibition to be stronger in women than in men. Thus, results of the current study provide evidence that the APM scales can discriminate in theoretically consistent ways between normal range personality variables. APM Meanness had a small, but significant positive association with Neuroticism. However, that association became nonsignificant when controlling for Disinhibition. Poy et al. (2014) reported very similar correlations between Meanness and Neuroticism in men and women to what was found in the current study, which appeared in the Poy et al. study to result from the contributions of the Angry Hostility and Impulsiveness facets of the Neuroticism domain. The BFI assesses FFM personality domains but not facets. Thus, it cannot be determined whether the small, significant association between Meanness and Neuroticism in the current study was influenced by Angry Hostility and Impulsiveness.

\section{General Discussion}

The current studies provide preliminary evidence of convergent, discriminant, and criterion validity of the Abbreviated Psychopathy Measure, a brief inventory that is closely modeled on the Triarchic Psychopathy Measure. Study 1 found that the APM Boldness, Meanness, and Disinhibition scales had high internal consistency reliabilities and were highly correlated with their counterpart scales on the TriPM. The APM Total score was very highly correlated with the TriPM Total score. APM Disinhibition had a very robust relationship with a measure of Antisocial Intent. Meanness and Disinhibition on both the APM and the TriPM were highly intercorrelated. For the TriPM, the magnitude of this correlation was higher in comparison to other published reports, albeit, several studies (Kyranides et al., 2017; Poy et al., 2014) reported associations between Meanness and Disinhibition that were close to the level found in this study. As noted earlier, the overlap between the TriPM Meanness and Disinhibition scales calls into question whether these scales can discriminate between related but distinct constructs. This question applies as well to the APM Meanness and Disinhibition scales. 
The APM appeared to depart most pointedly from the TriPM with respect to the Boldness scale, despite the APM and TriPM Boldness scales being well correlated. APM Boldness demonstrated moderately overlapping relationships with the APM Meanness and Disinhibition scales. As such, there is question whether the APM scales represent distinct constructs. However, as demonstrated in Study 2, when examined for its differential associations with normal range personality variables associated with psychopathy, the APM demonstrated a pattern that is similar to what has been demonstrated with the TriPM and normal range personality variables. APM Boldness had a robust association with Extraversion, while its modest, inverse association with Neuroticism increased when controlling for Disinhibition. As seen in studies with the TriPM (e.g., Stanley et al., 2013), APM Boldness also had a modest, but significant, positive relationship with Openness. APM Meanness had a strong, negative association with Agreeableness while demonstrating a more modest, inverse association with Conscientiousness. Miller and Lynam (2015) assert that the FFM domain of Agreeableness, i.e., extremely low agreeableness along with very high personal antagonism, is most essential to the construct of psychopathy. Thus, the APM Meanness scale appeared to strongly index this interpersonal antagonism feature of psychopathy. APM Disinhibition had a strong, inverse association with Conscientiousness and a more moderate, negative association with Agreeableness. The modest association between Disinhibition and Neuroticism increased when controlling for Boldness. Meanness had a modest, positive association with Neuroticism which may have been attributable to Angry Hostility (Patrick \& Drislane, 2014; Poy et al., 2014), albeit that could not be determined in the present study. In addition to high interpersonal antagonism, Miller and Lynam contend that a personality description of psychopathy derived from the FFM would include low levels of Conscientiousness (inhibition/constraint), a complex mixture of low and high levels of Neuroticism (i.e., low anxiety, depression, self-consciousness, and vulnerability, with high levels of angry hostility and impulsiveness), and high and low levels of Extraversion (high assertiveness and excitement seeking but low warmth). Personality dimensions consistent with Miller and Lynam's description of psychopathy can be regarded as consistent with higher levels of Boldness, Meanness, and Disinhibition on the APM. Higher levels of APM Boldness, Meanness, and Disinhibition were associated with higher levels of Extraversion, lower levels of Agreeableness (higher antagonism), lower levels of Conscientiousness, and modestly lower and higher levels of Neuroticism associated with Boldness and Disinhibition, respectively.

As suggested earlier, the APM Boldness scale appears to potentially tap into a more maladaptive or less socially favored quality of Boldness relative to the Boldness construct as indexed by the TriPM. There may be a greater emphasis on low harm avoidance or fearlessness in the APM in comparison to the TriPM. There may be some drift between low harm avoidance toward diminished self-restraint which may explain the strength of the relationship seen between APM Boldness and Disinhibition. Factor analytic study would be needed to clarify the latent structure of the APM. Still, like its counterpart on the TriPM, APM Boldness was found to be associated with positive traits of extraversion and openness. Interestingly, three of the four APM Boldness items that were endorsed by most respondents in the direction of higher boldness appear to relate to social potency. These items may have a somewhat weighted effect in accounting for associations between APM Boldness and positive traits. It is possible that APM Boldness and Disinhibition share a relationship with other co-related variables, e.g., boredom susceptibility, sensation seeking. Support for this can be seen in the finding by Brislin et al. (2015) that the MPQ-Triarchic Boldness and Disinhibition scales both were unique predictors of overall sensation seeking. MPQ-Tri Boldness and Disinhibition scales contributed significantly in regression analyses to the prediction of scores on measures of boredom susceptibility and disinhibition. Similarly, Miller and Lynam (2012) found that both the Fearless Dominance and Self-Centered Impulsivity factors of the PPI were moderately positively related to sensation seeking.

From the perspective of researchers who have not found evidence that Fearless Dominance or Boldness are essential to the construct of psychopathy (e.g., Gatner et al., 2016; Miller \& Lynam, 2012), APM Boldness, which was found to be moderately positively associated with a measure of Antisocial Intent and with the Egocentric and Antisocial subscales of the three factor LSRP, may offer an advantage over TriPM Boldness. That is, if boldness is an essential component of psychopathy, then it should predict negative outcomes including externalizing psychopathology. Indeed, Gatner et al. (2016) suggest it may be useful to adjust the TriPM Boldness scale to capture more maladaptive features. On the other hand, APM Boldness did not have a significant negative relationship with Agreeableness; thus, it did not index antagonistic features, as measured with the BFI, which, as suggested by Gatner et al., would be a useful if not validating feature of Boldness within the psychopathy construct.

The current study suggests the need to further examine the TriPM Boldness scale as approximately half of the items may reflect more positive, or socially favored content and half of the items may reflect more negative, or 
socially non-favored content, irrespective of reverse-coded items. Items reflecting positive or socially favored content may relate to self-confidence, self-esteem, and emotional resiliency, while those reflecting negative content may relate to tolerance for fear or anxiety-provoking situations and for potential harm or danger. It is possible that some of the content areas within TriPM Boldness, such as optimism and self-assurance, are endorsed more frequently across different groups, than other content areas such as intrepidness and tolerance for uncertainty. Some empirical support was found for this idea in that in the sample studied here (and in a previously examined larger sample), a subset of items designated as Bold Adaptive had a significantly higher mean score in comparison to the subset of items designated as Bold Maladaptive. The complex nature of the TriPM Boldness items may help explain why Boldness displays both adaptive and maladaptive correlates. It is possible that a more maladaptive component of TriPM Boldness has contributed to positive associations with external variables such as Erratic Lifestyle and Callous Affect (Drislane et al., 2014). It is also possible that a more adaptive, positive, socially favored component of Boldness may contribute to the apparent orthogonal relationship between TriPM Boldness and Disinhibition. Further study is needed to determine whether findings from the current study extend to other samples.

It was noted that men scored higher than women on the APM scales, on the TriPM scales, and on the Antisocial Intent scale. It has been reported (Skeem et al., 2011) that researchers generally agree that men display higher levels of psychopathy compared to women, and this has been observed with various psychopathy measures. Thus, the present findings were consistent with the literature on psychopathy.

This study also found that age was significantly and negatively associated with all the APM scales, with the TriPM scales except for Boldness, and with the Antisocial Intent scale, although effect sizes were of small magnitudes. Some evidence has been reported of age-related reduction of social deviance, antisocial behavior and criminal offenses tin psychopathic criminal offenders (Harpur \& Hare, 1994; Porter, Birt, \& Boer, 2001), although personality features such as callous, manipulative, egocentric traits might not similarly decline with age. The current study findings may be viewed as consistent with the above in that age did not have a strong, inverse relationship with psychopathic personality traits and antisocial intent as reflected in the measures used.

\subsection{Limitations and Future Directions}

A main limitation to consider in interpreting the findings of these studies is that self-report questionnaires were used which increases the possibility for shared method variance artificially inflating correlations among scale scores. Also, there was no control for any potential order effects. Both samples were not very large samples and both samples were obtained from the same source, i.e., MTurk, which limits generalizability to other settings. It may be beneficial to extend study of the APM to other populations and settings, including forensic settings. The current studies are clearly preliminary and additional study is needed to examine the relationship between the APM scales and relevant external correlates. Especially important will be to determine whether the APM scales assess distinctive aspects of psychopathy as well as other external correlates. A more nuanced examination of the relationship between the APM scales and the FFM may be conducted with use of a more comprehensive FFM measure such as the NEO-PI-R (Costa \& McCrae, 1992). APM Boldness should be examined in relation to maladaptive outcomes, e.g., aggression, antisocial behavior, various psychological variables, such as anxiety, positive and negative affect, as well as positive adjustment indicators. In comparison to the TriPM, the APM has a smaller percentage of items that are reverse-coded. This may make it more difficult to detect random responding as well as under- or over-reporting. The author developed a subscale of seven items that can be distributed among the three APM scales that may help detect socially desirable responding. It may be beneficial to include this subscale in future studies, although it would marginally increase the length of the APM.

\section{References}

Andershed, H., Kerr, M., Stattin, H., \& Levander, S. (2002). Psychopathic traits in non-referred youths: A new assessment tool. In E. Blaauw, \& L. Sheridan (Eds.), Psychopaths: Current international perspectives (pp. 131-158). The Hague: Elsevier.

Blagov, P.S., Patrick, C.J., Oost, K.M., Goodman, J.A., \& Pugh, A.T. (2015). Triarchic Psychopathy Measure: Validity in relation to normal-range traits, personality pathology, and psychological maladjustment. Journal of Personality Disorders, 30, 71-81. https://doi.org/10.1521/pedi_2015_29_182

Brislin, S.J., Drislane, L.E., Smith, S.T., Edens, J.F., \& Patrick, C.J. (2015). Development and validation of triarchic psychopathy scales from the Multidimensional Personality Questionnaire. Psychological Assessment, 27, 838-851. http://dx.doi.org/10.1037/pas000087 
Burisch, M. (1984). Approaches to personality inventory construction: A comparison of methods. American Psychologist, 39, 214-227. https://doi.org/10.1037/0003-066X.39.3.214

Burisch, M. (1997). Test length and validity revisited. European Journal of Personality, 18, 303-315. http://dx.doi.org/10.1002/(SICI)1099-0984(199711)11:4<303::AID-PER29273.0.CO;2\#

Christian, E., \& Sellbom, M. (2016). Development and validation of an expanded version of the three-factor Levenson Self-Report Psychopathy Scale. Journal of Personality Assessment, 98, 155-168. https://doi.org/10.1080/00223891.2015.1068176

Cleckley, H. (1941). The mask of sanity. St. Louis, MO: Mosby.

Cleckley, H. (1976). The mask of sanity (5 $5^{\text {th }}$ ed.). St. Louis, MO: Mosby.

Cooke, D.J., Hart, S.D., Logan, C., \& Michie, C. (2012). Explicating the construct of psychopathy: Development and validation of a conceptual model, the Comprehensive Assessment of Psychopathic Personality (CAPP). International Journal of Forensic Mental Health, 11, 242-252. https://doi.org/10.1080/14999013.2012.746759

Cooke, D.J., \& Michie, C. (2001). Refining the construct of psychopathy: Towards a hierarchical model. Psychological Assessment, 13, 171-188. https://doi.org/10.1037//1040-3590.13.2.171

Costa, P.T., Jr., \& McCrae, R.R. (1992). Revised NEO Personality Inventory (NEO-PI-R) and NEO Five-Factor Inventory (NEO-FFI) professional manual. Odessa, FL: Psychological Assessment Resources.

Drislane, L.E., Brislin, S.J., Kendler, K.S., Andershed, H., Larsson, H., \& Patrick. C.J. (2015). A triarchic model analysis of the Youth Psychopathic Traits Inventory. Journal of Personality Disorders, 29, 15-41. https://doi.org/10.1521/pedi_2014_28_144

Drislane, L.E., Patrick, C.J., \& Arsal, G. (2014). Clarifying the content coverage of differing psychopathy inventories through reference to the Triarchic Psychopathy Measure. Psychological Assessment, 26, 350-362. https://doi.org/10.1037/a0035152

Eisenbarth, H., Lilienfeld, S.O., \& Yarkoni, T. (2015). Using a genetic algorithm to abbreviate the Psychopathic Personality Inventory-Revised (PPI-R). Psychological Assessment, 27, 194-202. https://doi.org/10.1037/pas0000032

Evans, L., \& Tully, R. (2016). The Triarchic Psychopathy Measure (TriPM): Alternative to the PCL-R? Aggression and Violent Behavior, 27, 79-86. https://doi.org/10.1016/j.avb.2016.03.004

Fanti, K.A, Kyranides, M.N., Drislane, L.E., Colins, O.F., \& Andershed, H. (2016). Validation of the Greek Cypriot translation of the Triarchic Psychopathy Measure. Journal of Personality Assessment, 98, 146-154. https://doi.org/10.1080/00223891.2015.1077452

Gatner, D.T., Douglas, K.S., \& Hart, S.D. (2016). Examining the incremental and interactive effects of boldness with meanness and disinhibition within the triarchic model of psychopathy. Personality Disorders: Theory, Research, and Treatment, 7, 259-268. https://doi.org/10.1037/per0000182

Gatner, D.T., Douglas, K.S., \& Hart, S.D. (2017). Comparing the lexical similarity of the triarchic model of psychopathy to contemporary models of psychopathy. Journal of Personality. Advance online publication. https://doi.org/10.1111/jopy.12337

Hall, J.R., Drislane, L.E., Patrick, C.J., Morano, M., Lilienfeld, S.O., \& Poythress, N.G. (2014). Development and validation of triarchic construct scales from the Psychopathic Personality Inventory. Psychological Assessment, 26, 447-461. https://doi.org/10.1037/a0035665

Hare, R.D. (1991). The Hare Psychopathy Checklist-Revised. Toronto, Ontario, Canada: Multi-Health Systems.

Hare, R.D. (2003). The Hare Psychopathy Checklist-Revised (2 ${ }^{\text {nd }}$ ed.). Toronto, Ontario, Canada: Multi-Health Systems.

Hare, R.D., \& Neumann, C.S. (2008). Psychopathy as a clinical and empirical construct. Annual Review of Clinical Psychology, 4, 217-246. https://doi.org/10.1146/annurev.clinpsy.3.022806.091452

Harpur, T.J., \& Hare, R.D. (1994). Assessment of psychopathy as a function of age. Journal of Abnormal Psychology, 103, 604-609. https://doi.org/10.1037/0021-843X.103.4.604

John, O.P., Donahue, E.M., \& Kentle, R.L. (1991). The Big Five Inventory-Versions 4a and 54. Berkeley, CA: University of California, Berkeley, Institute of Personality and Social Research. 
John, O.P., \& Srivastava, S. (1999). The Big Five trait taxonomy: History, measurement, and theoretical perspectives. In L.A. Pervin \& O.P. John (Eds.), Handbook of personality: Theory and research $\left(2^{\text {nd }}\right.$ ed., pp. 102-138). New York, NY: Guilford.

Krueger, R.F., Markon, K.E., Patrick, C.J., Benning, S.D., \& Kramer, M. (2007). Linking antisocial behavior, substance use, and personality: An integrative quantitative model of the adult externalizing spectrum. Journal of Abnormal Psychology, 116, 645-666. https://doi.org/10.1037/0021-843X.116.4.645

Kyranides, M.N., Fanti, K.A., Sikki, M., \& Patrick, C.J. (2017). Triarchic dimensions of psychopathy in young adulthood: Associations with clinical and physiological measures after accounting for adolescent psychopathic traits. Personality Disorders: Theory, Research, and Treatment, 8, 140-149. https://doi.org/10.1037/per0000193

Levenson, M.R., Kiehl, K.A., \& Fitzpatrick, C.M. (1995). Assessing psychopathic attributes in a noninstitutionalized population. Journal of Personality and Social Psychology, 68, 151-158. https://doi.org/10.1037/0022-3514.68.1.151

Lilienfeld, S.O., \& Fowler, K.A. (2006). The self-report assessment of psychopathy: Problems, pitfalls, and promises. In C.J. Patrick (Ed.), Handbook of psychopathy (pp. 107-132). New York, NY: Guilford Press.

Lilienfeld, S.O., \& Widows, M.R. (2005). Psychopathic Personality Inventory-Revised (PPI-R) professional manual. Odessa, FL: Psychological Assessment Resources.

Marcus, D.K., Fulton, J.J., \& Edens, J.F. (2013). The two-factor model of psychopathic personality: Evidence from the psychopathic personality inventory. Personality Disorders: Theory, Research, and Tretment, 4, 67-76. https://doi.org/10.1037/a0025282

McCord, W., \& McCord, J. (1964). The psychopath: An essay on the criminal mind. Princeton, NJ: Van Nostrand.

Miller, J.D., \& Lynam, D.R. (2012). An examination of the Psychopathic Personality Inventory's nomological network: A meta-analytic review. Personality Disorders: Theory, Research, and Treatment, 3, 305-326. https://doi.org/10.1037/a0024567

Miller, J.D., \& Lynam, D.R. (2015). Psychopathy and personality: Advances and debates. Journal of Personality, 83, 585-592. https://doi.org/10.1111/jopy. 12145

Miller, J.D., Maples-Keller, J.L., \& Lynam, D.R. (2016). An examination of the three components of the Psychopathic Personality Inventory: Profile comparisons and tests of moderation. Psychological Assessment, 28, 692-710. https://doi.org/10.1037/pas0000221

Mills, J.F., Kroner, D.G., \& Forth, A.E. (2002). Measures of Criminal Attitudes and Associates (MCAA): Development, factor structure, reliability, and validity. Assessment, 9, 240-253. https://doi.org/10.1177/1073191102009003003

Mills, J.F., Kroner, D.G., \& Hemmati, T. (2004). The Measures of Criminal Attitudes and Associates (MCCA): The prediction of general and violent recidivism. Criminal Justice and Behavior, 31, 717-733. https://doi.org/10.1177/0093854804268755

O’Boyle, E.H., Forsyth, D.R., Banks, G.C., Story, P.A., \& White, C.D. (2015). A meta-analytic test of redundancy and relative importance of the dark triad and five-factor model of personality. Journal of Personality, 83, 644-664. https://doi.org/10.1111/jopy.12126

Paolacci, G., \& Chandler, J. (2014). Inside the Turk: Understanding Mechanical Turk as a participant pool. Current Directions in Psychological Science, 23, 184-188. https://doi.org/10.1177/0963721414531598

Patrick, C.J. (2010). Operationalizing the triarchic conceptualization of psychopathy: Preliminary description of brief scales for assessment of boldness, meanness, and disinhibition (Unpublished manual). Tallahassee, FL: Department of Psychology, Florida State University.

Patrick, C.J., \& Drislane, L.E. (2014). Triarchic model of psychopathy: Origins, operationalizations, and observed linkages with personality and general psychopathology. Journal of Personality, 83, 627-643. https://doi.org/10.1111/jopy.12119

Patrick, C.J., Fowles, D.C., \& Krueger, R.F. (2009). Triarchic conceptualization of psychopathy: Developmental origins of disinhibition, boldness, and meanness. Development and Psychopathology, 21, 913-938. https://doi.org/10.1017/S0954579409000492 
Porter, S., Birt, A.R., \& Boer, D.P. (2001). Investigation of the criminal and conditional release profiles of Canadian federal offenders as a function of psychopathy and age. Law and Human Behavior, 25, 647-661. https://doi.org/10.1023/A:1012710424821

Poy, R., Segarra, P., Esteller, A., Lopez, R., \& Molto, J. (2014). FFM description of the triarchic conceptualization of psychopathy in men and women. Psychological Assessment, 26, 69-76. https://doi.org/10.1037/a0034642

Saucier, G.D. (1994). Mini-markers: A brief version of Goldberg's unipolar Big-Five Markers. Journal of Personality Assessment, 63, 506-516. https://doi.org/10.1207/s15327752jpa6303_8

Seibert, L.A., Miller, J.D., Few, L.R., Zeichner, A., \& Lynam, D.R. (2010). An examination of the structure of self-report psychopathy measures and their relations with general traits and externalizing behaviors. Personality Disorders: Theory, Research, and Treatment, 2, 193-208. https://doi.org/10.1037/a0019232

Sellbom, Lilienfeld, S.O., Fowler, K., \& McCrary, K.L. (in press). The self-report assessment of psychopathy: Challenges, pitfalls, and promises. In C.J. Patrick (Ed.), Handbook of psychopathy $\left(2^{\text {nd }}\right.$ ed.). New York, NY: Guilford Press.

Sellbom, M., \& Phillips, T.R. (2013). An examination of the triarchic conceptualization of psychopathy in incarcerated and nonincarcerated samples. Journal of Abnormal Psychology, 122, 208-214. https://doi.org/10.1037/a0029306

Semel, R.A. (2016). The Caring-Uncaring Emotional (CUE) Inventory: A pilot study of a new measure of affective psychopathy traits. International Journal of Psychological Studies, 8, 1-15. http://dx.doi.org/10.5539/ijps.v8n4p1

Semel, R.A. (2017). Examining the Caring-Uncaring Emotional (CUE) Inventory as a measure of affective, callous-unemotional psychopathy traits in a community sample of adults. International Journal of Psychological Studies, 9, 35-50. http://doi.org/10.5539/ijps.v9n3p35

Shou, Y., Sellbom, M., \& Xu, J. (2017). Psychometric properties of the Triarchic Psychopathy Measure: An item response theory approach. Personality Disorders: Theory, Research, and Treatment. Advance online publication. http://dx.doi/org/10.1037/per0000241

Skeem, J.L., Polaschek, D.L.L., Patrick, C.J., \& Lilienfeld, S.O. (2011). Psychopathic personality: Bridging the gap between scientific evidence and public policy. Psychological Science in the Public Interest, 12, 95-162. https://doi.org/10.1177/1529100611426706

Somma, A., Borroni, S., Drislane, L.E., \& Fossati, A. (2016). Assessing the triarchic model of psychopathy in adolescence: Reliability and validity of the Triarchic Psychopathy Measure (TriPM) in three samples of Italian community-dwelling adolescents. Psychological Assessment, 28, e36-e48. https://doi.org/10.1037/pas0000184

Stanley, J.H., Wygant, D.B., \& Sellbom, M. (2013). Elaborating on the construct validity of the Triarchic Psychopathy Measure in a criminal offender sample. Journal of Personality Assessment, 95, 343-350. https://doi.org/10.1080/00223891.2012.735302

Tellegen, A. (2003). Multidimensional Personality Questionnaire. Minneapolis, MN: University of Minnesota Press.

Van Dongen, J.D.M., Drislane, L.E., Nijman, H., Soe-Agnie, S.E., \& Van Marle, H.J.C. (2017). Further evidence for reliability and validity of the Triarchic Psychopathy Measure in a forensic sample and a community sample. Journal of Psychopathology and Behavioral Assessment, 39, 58-66. https://doi.org/10.1007/s10862-016-9567-5

Wall, T.D., Wygant, D.B., \& Sellbom, M. (2015). Boldness explains a key difference between psychopathy and antisocial personality disorder. Psychiatry, Psychology and Law, 22, 94-105. https://doi.org/10.1080/13218719.2014.919627

Widiger, T.A., \& Lynam, D.R. (1998). Psychopathy and the five-factor model of personality. In T. Millon, E. Simonsen, M. Birket-Smith, \& R.D. Davis (Eds.), Psychopathy: Antisocial, criminal, and violent behavior (pp. 171-187). New York, NY: Guilford Press.

Williams, K.M., Paulhus, D.L., \& Hare, R.D. (2007). Capturing the four-factor structure of psychopathy in college students via self-report. Journal of Personality Assessment, 88, 205-219. https://doi.org/10.1080/00223890701268074 


\section{Notes}

1 These included TriPM Boldness items 1, 3, 4, 6, 7, 8, 9, 11, 13, 15, 17, 19

2 Results are from a previous dataset $(N=316)$ obtained by this author in which a more preliminary form of the APM was compared with the TriPM. In that dataset, TriPM Bold Adaptive items, i.e., items that were endorsed by the majority of respondents in the direction of being higher on the dimension of Boldness, were items $1,3,4$, $6,8,9,11,17,19$. Bold Maladaptive items, i.e., items that are higher on the dimension of Boldness and were endorsed by a minority of respondents, were items $2,5,7,10,12,13,14,15$. Items 16 and 18 were evenly split. Also, for $68 \%$ of the TriPM Boldness items, the response with the single highest frequency was in the keyed direction of being higher on the dimension of Boldness.

\section{Copyrights}

Copyright for this article is retained by the author(s), with first publication rights granted to the journal.

This is an open-access article distributed under the terms and conditions of the Creative Commons Attribution license (http://creativecommons.org/licenses/by/4.0/). 